\title{
Defining the elusive boundaries of chronic active Epstein-Barr virus infection
}

\author{
Sebastian Fernandez-Pol, Oscar Silva and Yasodha Natkunam \\ Department of Pathology, Stanford University School of Medicine, CA, USA
}

E-mail:yaso@stanford.edu

doi:10.3324/haematol.2018.193714

T he Epstein-Barr virus (EBV) infects more than $90 \%$ of people by early adulthood. While childhood EBV infection tends to be asymptomatic, adolescents and young adults often develop infectious mononucleosis (IM). With rare exceptions, IM usually resolves spontaneously. EBV is also implicated in the development of several hematolymphoid malignancies derived from B-, T- and natural killer (NK)-cells, including Burkitt lymphoma, classic Hodgkin lymphoma, extranodal NK-/T-cell lymphoma, nasal type (ENKTL), and immunodeficiency-associated lymphoproliferative disorders (LPD). ${ }^{1.5}$

Primarily because of their rarity, systemic $\mathrm{EBV}^{+} \mathrm{T}$-cell and NK-cell LPDs are not completely understood. Criteria to separate clinical risk groups at diagnosis are confounded by several important factors: 1) non-specific histopathological and immunophenotypic features that overlap with infectious and inflammatory conditions as well as with other $\mathrm{T}$ - and NK-LPDs; 2) lack of markers to reliably predict clinical behavior that ranges from self-limiting proliferations to those that are rapidly fatal; 3) molecular clonality, although often detected, is not necessarily indicative of malignancy; and 4) inconsistent association with hemophagocytic lymphohistiocytosis (HLH), whose presence is often life-threatening. ${ }^{1-3,6-11}$ Large, clinically well-annotated cohorts of patients offer a tremendous opportunity to investigate rare subtypes of $\mathrm{EBV}^{+} \mathrm{T}$ - and NK-cell proliferations, define diagnostic and prognostic criteria, and explore disease boundaries. To that end, the paper by Kawamoto et al. in this issue of Haematologica addresses a rare type of $\mathrm{EBV}^{+} \mathrm{T}$ - and NK-cell proliferation in adult patients with chronic active EBV-like features (adult-onset CAEBV). ${ }^{12}$

Chronic active EBV is characterized by $\mathrm{EBV}^{+} \mathrm{T}$ - or NK-cell proliferations and includes systemic as well as cutaneous forms. Systemic CAEBV presents with fever, lymphadenopathy and splenomegaly, and typically develops in immunocompetent patients following primary EBV infection. The initial phase resembles an IM-like illness. These lymphoid proliferations may be polyclonal, oligoclonal or monoclonal, and have a propensity to evolve into a systemic $\mathrm{EBV}^{+} \mathrm{T}$ - or NK-cell lymphoma. CAEBV most often occurs in children and adolescents without a history of immunodeficiency or autoimmunity. The clinical course and prognosis of CAEBV is highly variable: while some patients exhibit a protracted disease course, others experience a fulminant form of the disease accompanied by HLH. ${ }^{1-3,8-10}$

Chronic active EBV of T- and NK-cell types have a strong ethnic predisposition, and are most frequent in East Asia and in indigenous populations of Central and South America. ${ }^{1,6-11}$ It is rare in Western and African populations. ${ }^{1,13}$ The etiology of CAEBV is unknown, although susceptibility is thought to result from defective cytotoxic T-cell or NK-cell activity against EBV-infected cells. In addition to a defective host immune response, EBV viral load is a key factor that impacts the severity of the disease. ${ }^{1,2}$ Rare CAEBV cases with $\mathrm{EBV}^{+} \mathrm{B}-$ cell proliferations have also been reported in Asia and in the United States. ${ }^{2,13}$ Although CAEBV typically affects children and adolescents, rare cases of adult-onset CAEBV have been reported; these are associated with a worse prognosis. ${ }^{1,2}$

Kawamoto et al. describe a cohort of 54 patients with adult-onset CAEBV (defined as onset $>15$ years of age). ${ }^{12}$ For comparison, they utilized control groups of patients including pediatric onset CAEBV $(n=75)$, and ENKTL of nasal $(n=37)$ and non-nasal $(n=45)$ types. The diagnostic criteria for CAEBV as defined in the recently revised World Health Organization classification include persistent IM-like symptoms for more than three months, increased EBV DNA $\left(>10^{2.5}\right.$ copies $/ \mathrm{mg}$ ) in peripheral blood, histological evidence of organ disease, and EBV RNA or viral protein in affected tissues. ${ }^{1}$ All patients in the Kawamoto et al. study met these criteria. Patients with prior immunodeficiency, including HIV infection, were rigorously excluded. A median age of onset at 39 years (range 16-86 years) and the period from estimated onset to diagnosis of over one year typified this cohort. In addition, there was a bimodal age distribution for adult-onset CAEBV, a finding that had not been previously appreciated.

The EBV+ lymphoid infiltrates of CAEBV most often lack atypical features and may mimic non-specific inflammatory or infectious conditions. As such, a strong index of suspicion is necessary to perform appropriate testing for EBV DNA in peripheral blood and EBV RNA (in situ hybridization for EBER) in affected tissue to establish a diagnosis. T-cell CAEBV patients typically have high titers of IgG antibodies against EBV viral capsid antigen and early antigen, and demonstrate a worse prognosis when compared to NK-cell type CAEBV..$^{1.3}$ In the Kawamoto et al. study, ${ }^{12}$ EBV DNA viral load was measured in peripheral blood as well as in tissue by in situ hybridization for EBER RNA. Lymphadenopathy was more frequent in patients with T-cell CAEBV, while skin involvement was more frequent in NKcell CAEBV. There were no significant differences in outcome between the T-cell and NK-cell cases among adultonset CAEBV patients. When patients aged over 50 years were analyzed separately, no significant differences were found in comparison to all adult-onset CAEBV cases.

Cutaneous CAEBV includes hydroa vacciniforme-like lymphoproliferative disorder (HV-LPD), which typically occurs in children. It is most often a cytotoxic $\mathrm{CD}^{+} \mathrm{T}$-cell proliferation, although CD4, CD56 and CD30 are positive in subsets of cases. The clinical course is variable, and the severity may depend on photosensitivity. ${ }^{1,29,14-16}$ Pathological diagnosis may be confounded by the lack of distinctive histological features. Clinical correlation and testing for EBV is paramount to making the diagnosis. Severe allergy to mos- 
quito bites is an NK-cell proliferation that results from local and systemic symptoms following a mosquito bite. Immunophenotypic features, particularly CD30 expression in a subset may overlap with features of lymphomatoid papulosis. ${ }^{1,2,9,17}$ Recognition and timely diagnosis is important because patients with severe allergy to mosquito bites may progress to systemic NK-cell type CAEBV, aggressive NK-cell leukemia, or ENKTL. ${ }^{17}$ In comparison to pediatric onset CAEBV, the adult cohort analyzed by Kawamoto et al. had significantly lower frequency of fever and greater frequency of skin lesions, including HV-LPD and severe allergy to mosquito bites. ${ }^{12}$

Distinguishing CAEBV from more aggressive $\mathrm{EBV}^{+} \mathrm{T}$ and NK-cell proliferations can be challenging, particularly given the propensity of CAEBV to progress to a more aggressive LPD. It is, however, important to recognize and accurately diagnose LPDs in this continuum because early intervention may offer the only solution for patients progressing toward a fulminant disease. Therefore, defining the boundary between CAEBV and more aggressive $\mathrm{EBV}^{+} \mathrm{T}$ - and NK-LPDs, and the development of guidelines for the management of such patients, are urgently needed. In the Kawamoto et al. study, although no specific markers of disease severity or progression were identified, thrombocytopenia (platelets $<100 \times 10^{9} / \mathrm{L}$ ), high EBNA titer $(\geq 40)$, and HLH at initial diagnosis were associated with a worse overall clinical outcome. HLH was diagnosed using the HLH 2004 guidelines and was more frequent $(46 \%)$ in the bone marrows of adult-onset CAEBV. Most importantly, allogeneic stem cell transplan-

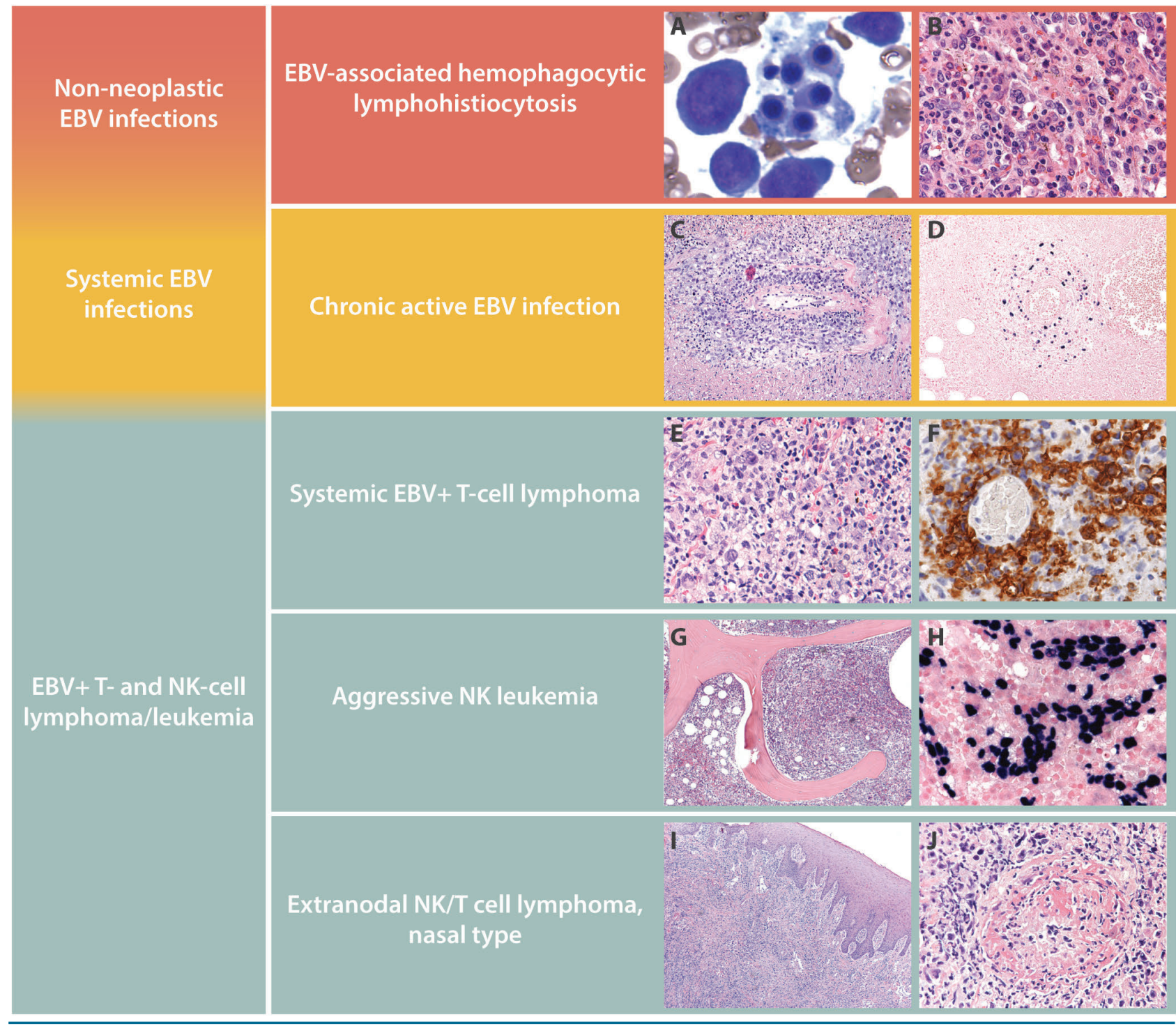

Figure1. Spectrum of Epstein-Barr virus-positive $\left(\mathrm{EBV}^{+}\right)$T-cell and natural killer (NK)-cell lymphoproliferative disorders (LPD). Characteristic features of EBV ${ }^{+}$T-cell and NK-cell LPD are shown. Hemophagocytic lymphohistiocytosis (HLH) in the bone marrow of a 3-year old Asian child with EBV infection. Core biopsy highlights HLH within bone marrow sinuses (A and B). Chronic active EBV infection causing an oral cavity lesion in an otherwise asymptomatic 14-year old Caucasian adolescent showing a polymorphous lymphoid infiltrate with $\mathrm{EBV}^{+}$cells in an angiocentric distribution (C and D). Systemic EBV T-cell lymphoma in an otherwise asymptomatic 8-year old child presenting with persistent fever and chills. A CD56 stain highlights the atypical infiltrate and underscores the importance of testing for EBV in a patient with unexplained fevers (E and F). Aggressive NK leukemia replacing the bone marrow in a 42-year old man who presented with pancytopenia and massive hepatosplenomegaly. EBER in situ hybridization shows an exuberant $\mathrm{EBV}^{+}$interstitial infiltrate in the bone marrow $(\mathrm{G}$ and $\mathrm{H})$. Extranodal NK/T-cell lymphoma, nasal type causing an ulcerated palatal lesion in a 51-year old man from Guatemala. Prominent necrosis and angiodestruction characterize the atypical lymphoid infiltrate (I and J). 
tation was found to be the most effective treatment choice for prolonged overall survival. ${ }^{12}$

Of particular interest in the study by Kawamoto et al. ${ }^{12}$ is their comparison of overall survival in patients with pediatric and adult-onset CAEBV with nasal and nonnasal ENKTL. Adult-onset CAEBV showed a worse overall survival in comparison to pediatric onset CAEBV and ENKTL. In fact, adult-onset CAEBV had an overall survival comparable to that of non-nasal ENKTL, which is generally more aggressive than its nasal counterpart.

In the pediatric age group, systemic $\mathrm{EBV}^{+} \mathrm{T}-\mathrm{cell}$ lymphoma of childhood, a de novo CD8 ${ }^{+} \mathrm{T}$-cell lymphoma with an aggressive clinical course, must be separated from CAEBV. ${ }^{1-3,8}$ One key feature that helps distinguish CAEBV from systemic EBV ${ }^{+}$T-cell lymphoma of child- hood is that the $\mathrm{T}$ cells in CAEBV are predominantly $\mathrm{CD}^{+}$and less frequently of cytotoxic CD8 origin. In addition, CAEBV can show clinical and histological overlap with aggressive NK-cell leukemia and ENKTL. Age of onset, together with immunophenotypic differences help in the diagnosis: $\mathrm{sCD}^{+}, \mathrm{CD} 56^{\circ}, \mathrm{CD}^{+}$and/or $\mathrm{CD}_{4}^{+}$in systemic $\mathrm{EBV}^{+} \mathrm{T}$-cell lymphoma of childhood; sCD3 , cCD3 ${ }^{+}$, $\mathrm{CD}^{+}{ }^{+}, \mathrm{CD}^{6} 6^{\mathrm{dim}}, \mathrm{CD} 8$ in aggressive NK-cell leukemia; and $\mathrm{CD}^{+}, \mathrm{CD} 5, \mathrm{CD}^{+} 6^{+/}, \mathrm{CCD}^{+}, \mathrm{EBER}$ in ENKTL. The presence of $\mathrm{T}$-cell clonality in systemic $\mathrm{EBV}^{+} \mathrm{T}$-cell lymphoma can further help distinguish that entity from aggressive NK-cell leukemia, which lacks T-cell clones. Markers associated with NK-cell differentiation, such as CD16, can also be useful, as CD16 is positive in $75 \%$ of aggressive NK leukemia but usually absent in ENKTL.

Table 1. Characteristic features of Epstein-Barr virus (EBV)-positive (EBV+) T-cell and natural killer (NK)-cell lymphoproliferative disorders.

\section{EBV-associated hemophagocytic lymphohistiocytosis (HLH)}

- Non-neoplastic proliferation typically seen in the pediatric age group

- Acute presentation without prior history of immunodeficiency or EBV infection

- Most common in patients of Asian descent

- T-cell monoclonality is often detected but is not an indication of malignancy

- Hyperbilirubinemia ( $>1.8 \mathrm{mg} / \mathrm{dL}$ ) and hyperferritinemia $(>20,300 \mathrm{ng} / \mathrm{mL})$ at diagnosis are poor prognostic factors

- HLH 2004 protocol is used for management; lack of response may necessitate bone marrow transplantation

- May be self-limiting in some cases

\section{Chronic active EBV (CAEBV) infection}

- Cutaneous and systemic forms exist, although symptoms can overlap

- Cutaneous CAEBV includes severe mosquito bite allergy and hydroa vacciniforme-like lymphoproliferative disorders

- Systemic CAEBV presents with fever, lymphadenopathy and splenomegaly, and typically follows primary EBV infection

- Most common in children and young adults

- Most common in patients of Asian or Hispanic descent

- Follows a chronic, relapsing clinical course with a risk of progression to systemic EBV+ T- or NK-cell lymphoma

- CAEBV of T-cell origin has high IgG titers against EBV viral capsid and early antigens and a worse prognosis

- Adult-onset CAEBV is associated with a worse prognosis

\section{Systemic EBV+ T-cell lymphoma}

- Acute, de novo T-cell lymphoma of $\mathrm{EBV}^{+}$cytotoxic T cells

- Most common in children and young adults

- Most common in Asians and in indigenous populations of Central and South America

- Most patients are immunocompetent

- Most patients develop HLH

- Follows a fulminant clinical course with poor response to chemotherapy and death within days to weeks of diagnosis

\section{Aggressive NK leukemia}

- Systemic neoplastic proliferation of NK cells frequently associated with EBV

- Most commonly involves peripheral blood, bone marrow, liver and spleen

- Most common in young to middle-aged adults with a median age of 40 years

- Most common in patients of Asian descent

- May evolve from CAEBV, particularly in younger patients

- Follows a fulminant clinical course with multi-organ failure and poor response to chemotherapy, with a median survival of $<2$ months

\section{Extranodal NK/T cell lymphoma, nasal type}

- Extranodal lymphoma of EBV+ cytotoxic T or NK cells associated with angiodestruction and prominent necrosis

- Affects immunocompetent as well as immunocompromised patients

- Most commonly involves the upper aerodigestive tract, skin, soft tissue, gastrointestinal tract and testes

- Most common in adults with a median age of 44-54 years

- Most common in Asians and in indigenous populations of Central and South America

- Clinical course is variable: some respond well to chemotherapy and up-front radiation while others succumb to disseminated disease

- Advanced stage, invasion of bone or skin, $\mathrm{EBV}^{+}$cells in the bone marrow and a high proliferation index are unfavorable prognostic factors

- Extranasal disease is highly aggressive and is refractory to treatment

- Some cutaneous cases may have a protracted clinical course

Nodal peripheral T-cell lymphoma, $\mathbf{E B V}^{+}$

- Other $\mathrm{EBV}^{+}$peripheral T-cell lymphomas (not further discussed in the report) 
Recently, recurring somatic mutations, including $D D X 3 X$, STAT3, STAT5B, JAK3, and TP53, have been identified in ENKTL. ${ }^{18-20}$ Mutational profiles in other subtypes of $\mathrm{EBV}^{+}$ T- and NK-cell LPDs will be necessary to translate these discoveries for diagnostic purposes. It is hoped that clinically well-annotated cohorts, such as that described by Kawamoto et al., will be used to define molecular profiles in future studies.

In summary, Kawamoto et al. present a comprehensive characterization of a rare $\mathrm{EBV}^{+} \mathrm{T}$ - and NK-cell LPD that occurs primarily in adults and has features of CAEBV..$^{12}$ As is typical of rare diseases, precise diagnostic criteria and classification can be challenging unless sufficient numbers of patients become available for study. By providing a relatively large cohort of patients with detailed clinical annotation, this paper serves to extend knowledge in the field, and raises the possibility that adult-onset CAEBV may be among the most aggressive of $\mathrm{EBV}^{+} \mathrm{T}$ - and NKcell LPDs. It remains to be seen if future molecular characterization of adult-onset CAEBV is likely to offer insights that will allow accurate and early diagnosis and lead to improved outcomes for these patients.

\section{References}

1 Swerdlow SH, Campo E, Harris NL, et al. WHO Classification of Tumours of Haematopoietic and Lymphoid Tissues. (4th ed.) IARC, Lyon, 2017.

2. Kimura H, Ito Y, Kawabe S, et al. EBV-associated T/NK-cell lymphoproliferative diseases in nonimmunocompromised hosts: prospective analysis of 108 cases. Blood. 2012;119(3):673-686.

3. Swerdlow SH, Jaffe ES, Brousset P, et al. Cytotoxic T-cell and NK-cell lymphomas: current questions and controversies. Am J Surg Pathol. 2014;38(10):60-71.

4. Natkunam Y, Gratzinger D, de Jong D, et al. Immunodeficiency and Dysregulation: Report of the 2015 Workshop of the Society for Hematopathology/European Association for Haematopathology. Am J Clin Pathol. 2017;147(2):124-128.

5. Gratzinger D, de Jong D, Jaffe ES, et al. T- and NK-Cell Lymphomas and Systemic Lymphoproliferative Disorders and the Immuno- deficiency Setting: 2015 SH/EAHP Workshop Report - Part 4. Am J Clin Pathol. 2017;147(2):188-203.

6. Kikuta H, Sakiyama, Y, Matsumoto S, et al. Fatal Epstein-Barr virus associated hemophagocytic syndrome. Blood. 1993;82(11):3259-3264.

7. Su IJ, Chen RL, Lin DT, et al. Epaterin-Barr virus (EBV) infects T lymphocytes in childhood EBV-associated hemophagocytic syndrome in Taiwan. Am J Pathol. 1994;144(6):1219-1225.

8. Quintanilla-Martinez L, Kumar S, Fend F, et al. Fulminant EBV(+) T-cell lymphoproliferative disorder following acute/chronic EBV infection: a distinct clinicopathologic syndrome. Blood. 2000;96(2):443-451.

9. Kimura H, Hoshino Y, Kanegane H, et al. Clinical and virologic characteristics of chronic active Epstein-Barr virus infection. Blood. 2001;98(2):280-286.

10. Suzuki K, Ohshima K, Karube K, et al. Clinicopathological states of Epsterin-Barr virus associated T/NK-cell lymphoproliferative disorders (severe chronic active EBV infection) of children and young adults. Int J Oncol. 2004;24(5):1165-1174.

11. Suzuki R, Suzumiya J, Nakamura S, et al. Aggressive natural killer-cell leukemia revisited: large granular lymphocyte leukemia of cytotoxic NK cells. Leukemia. 2004;18(4):763-770.

12. Kawamoto K Miyoshi H, SuzukiT, et al. A distinct subtype of Epstein Barr virus positive T/NK cell lymphoproliferative disorder: Adult patients with Epstein Barr virus infection-like features. Haematologica. 2018;103(6):1018-1028.

13. Cohen JI, Jaffe ES, Dale JK, et al. Characterization and treatment of chronic active Epstein-Barr virus disease: a 8-year expereince in the United States. Blood. 2011;117(22):5835-5849.

14. Iwatsuki K, Xu Z, Takata M, et al. The association of latent EpsteinBarr virus infection with hydroa vacciniforme. Br J Dermatol. 1999;140(4):715-721.

15. Gupta G, Man I, Kemmett D. Hydroa vacciniforme: A clinical and follow-up study of 17 cases. J Am Acad Dermatol. 2000;42(2 Pt 1):208213.

16. Quintanilla-Martinez L, Ridaura C, Nagl F, et al. Hydroa vacciniformelike lymphoma: a chronic EBV+ lymphoproliferative disorder with risk to develop a systemic lymphoma. Blood. 2013;122(18):3101-3110.

17. Kempf W, Kazakov D V, Schärer L, et al. Angioinvasive lymphomatoid papulosis: a new variant simulating aggressive lymphomas. Am J Surg Pathol. 2013;37(1):1-13.

18. Koo GC, Tan SY, Tang T, et al. Janus kinase 3-activating mutations identified in natural killer/T-cell lymphoma. Cancer Discov. 2012;2(7):591-597.

19. Küçük C, Jiang B, Hu X, et al. Activating mutations of STAT5B and STAT3 in lymphomas derived from $\gamma \delta-\mathrm{T}$ or NK cells. Nat Commun. 2015;6:6025.

20. Jiang L, Gu Z-H, Yan Z-X, et al. Exome sequencing identifies somatic mutations of DDX3X in natural killer/T-cell lymphoma. Nat Genet. 2015;47(9):1061-1066. 